\title{
conomics
}

The Open-Access, Open-Assessment E-Journal

Vol. 3, 2009-12 | April 9, 2009 | http://www.economics-ejournal.org/economics/journalarticles/2009-12

\section{Now or Never: Environmental Protection under Hyperbolic Discounting}

\author{
Ralph Winkler \\ Center of Economic Research at ETH Zurich
}

\begin{abstract}
The author analyzes the optimal investment in environmental protection in a model of non-overlapping hyperbolically discounting agents. He shows that, in the long run and in the absence of a commitment device, society is stuck in a situation where all agents prefer further investments, yet no agent invests. This holds no matter whether agents are aware of the time inconsistency of their preferences. As a consequence, awareness of the time-inconsistency problem poses at best a short run remedy. Moreover, such an outcome may be Pareto inefficient and may explain the weak performance of long-run environmental policies.
\end{abstract}

Special issue "Discounting the Long-Run Future and Sustainable Development”

JEL: D90, Q50, Q58

Keywords: Environmental policy; environmental protection; hyperbolic discounting, Markov perfect equilibria; time-inconsistency

\section{Correspondence}

Ralph Winkler, CER-ETH - Center of Economic Research at ETH Zurich, ZUE D4, CH-8092 Zurich, Switzerland, mail@ralph-winkler.de

The author is grateful to Fabio Antoniou, Stefan Baumgärtner, Vangelis Dyikitopoulos, Jürgen Eichberger, Malte Faber, Hans Gersbach, Larry Karp, Ulf Moslener, John Proops, Maik Schneider and Christian Traeger for valuable comments on an earlier draft. Financial support by the Deutsche Forschungsgemeinschaft (German Research Foundation) under the graduate programme "Environmental and Resource Economics" and by the European Commission under the Marie Curie Intra-European Fellowship scheme, No. MEIF-CT-2003-501536, is gratefully acknowledged. 


\section{Introduction}

A ubiquitous feature in environmental economics is that welfare costs and benefits of projects undertaken to mitigate environmental problems spread over decades or even centuries (e.g., global climate change, biodiversity loss, depletion of the ozone layer and disposal of radioactive waste). With the standard exponential discounting approach, first introduced by Samuelson (1937) and put on an axiomatic basis by Debreu (1954) and Koopmans (1960), outcomes in the far distant future are worth close to nothing for any positive discount rate. In many people's view this is not the way we do think or should think about the far distant future. Therefore, discounting has been a controversial topic, with proposals ranging from ad-hoc adjustments to alternative axiomatic derivations (Lind, 1982, Rabl, 1996, Portnev and Wevant, 1999, Heal, 1998).

One recent approach to deal with the shortcomings of exponential discounting is hyperbolic discounting, i.e., the discount rate is not constant but declining over time. It has been advocated for three reasons (for an overview see Pearce et al., 2003, Groom et al., 2005). First, empirical evidence suggests that decision makers use declining rather than constant discount rates (Gintis, 2000, Frederick et al., 2002). Second, uncertainty over the future state of the world leads to declining certainty-equivalent discount rates (Weitzman, 1998, Azfar, 1999, Gollier, 2002). Third, declining discount rates are consistent with a rule, which balances the welfare of current and future generations (Chichilniskv, 1996, Li and Löfgren, 2000).

In this paper, we analyze the optimal investment in environmental protection, given a hyperbolically discounting society, which consists of a series of non-overlapping generations, each represented by a single agent. To capture the common pattern of many environmental problems, we assume that the present generation faces the costs of investment, while the benefits spread over all subsequent generations.

It is well known from the literature that hyperbolic discounting bears the problem of time-inconsistency. As Strotz (1956) has pointed out, this implies that an ex ante optimal decision is not carried out, because a later re-evaluation suggests that it is not optimal anymore. Although the time-inconsistency property of hyperbolic discounting has been used to model 'irrational' behavior, such as addiction and procrastination (Ackerlof, 1991, O'Donoghue and Rabin, 1999, Brocas and Carrillo, 2001, Gruber and Koszegi, 2001), there is a debate on how serious is the problem of time-inconsistency in long-term and intergenerational decision making (Heal, 1998, Henderson and Bateman, 1995, Pearce et al., 2003). In fact, if declining discount rates stem from uncertainty over future states of the world there is no issue of time-inconsistency if plans are updated as soon as better information is availbale. If, however, declining discount rates are due to declining pure rates of time preference, either because of imperfect altruism towards future generations or due to ethical considerations balancing the welfare of present and future generations, time-inconsistency has to be taken seriously.

Assuming hyperbolically discounting agents, we compare their investment decisions under three different behavioral patterns. Naive agents do not recognize that their preferences are non-stationary. Thus, they do not anticipate that subsequent agents will not stick to their ex ante optimal plan. If agents are aware of the 
time-inconsistency problem, they are either committed if the first agent can commit all subsequent agents to her ex ante optimal plan, or are called sophisticated if no commitment mechanism is availbale. Then, time-consistent planning is equivalent to a non-cooperative sequential investment game all agents play against each other (Phelps and Pollak, 1968). We show that, although it is ex ante optimal to do so, neither naive nor sophisticated agents invest in later periods but not in the first period. Without a commitment device society is eventually stuck in a situation where all agents prefer further investments in the long run, yet no agent will invest. As this holds for both naive and sophisticated agents, awareness of the time-inconsistency problem may only pose a short run remedy. Moreover, such an outcome may be Pareto inefficient and may explain why the performance of long-run environmental policies is weak.

The paper is organized as follows. In section 2 the model is introduced. The ex ante optimal plan is analyzed in section 3, while section 4 is devoted to the ex post outcome. In section [5, we examine some of our model assumptions and discuss implications for environmental policy. Section $[$ concludes.

\section{A Simple Model of Environmental Protection}

Consider the following situation: Society can invest in a project that is aimed to decrease the impact of the society's economic activity on the natural environment. We call this project environmental protection. Environmental protection in period $t, k_{t}$, is assumed to be a capital good, i.e., investments $i_{t}$ in different periods $t$ accumulate over time. As we focus on long-run environmental problems, we consider environmental protection to be long lasting, and thus abstract from depreciation. Then, the equation of motion for environmental protection is given by:

$$
k_{t+1}=k_{t}+i_{t} .
$$

For given $k_{0}$ this implies that $k_{t}=k_{0}+\sum_{\tau=0}^{t} i_{\tau}$. Investments $i_{t}$ in environmental protection are assumed to be sunk, i.e., de-investment is not possible and, thus, $i_{t} \geq 0$ holds for all periods $t$. To rule out solutions with ever increasing stocks of environmental protection we assume an arbitrary but finite upper bound of environmental protection 1 Without loss of generality we scale environmental protection such that $k(t) \in[0, \bar{k}], t \geq 0$, where $\bar{k}$ denotes the arbitrary but finite upper bound.

Society's payoff of environmental protection in period $t$ is given by $P\left(i_{t}, k_{t}\right)$, which is a strictly concave function in both arguments (partial derivatives are indicated by subscripts throughout the paper: $\left.P_{i i}<0, P_{k k}<0, P_{i i} P_{k k}-P_{i k}^{2}>0\right)$. A central assumption in this model is that utility costs and benefits of investments in environmental protection do not accrue at the same time. We assume that $P$ is strictly decreasing in $i_{t}\left(P_{i}<0\right)$ and strictly increasing in $k_{t}\left(P_{k}>0\right)$. This implies that costs (investments in environmental protection $i_{t}$ ) occur before the benefits (stock of environmental protection $k_{t}$ ), as investments today accumulate the stock of the next period 2 In addition, we assume that the marginal costs of investment are non-

\footnotetext{
${ }^{1}$ In fact, this assumption rules out Ponzi game solutions in case of sophisticated agents.

${ }^{2}$ Examples include the disposal of nuclear waste and the abatement of $\mathrm{CO}_{2}$ to slow down the
} 
decreasing with the level of environmental protection, i.e., there are no economies of scale in environmental protection $\left(P_{i k} \leq 0\right)$. This amounts to the assumption that cheap options to enhance environmental quality are chosen first and, thus, marginal costs increase with the level of environmental protection.

We assume an infinite series of non-overlapping "generations", each the length of one period. As an example, think of these generations as the succession of ruling governments in a representative democracy with a period length equal to the election period. In each period $t$, there is a decision maker, in the following called agent $t$, who is in charge of the investment decision $i_{t}$ in environmental protection in period $t$. Each agent $t$ cares about current and future payoffs, but treats past decisions as bygone. All agents are supposed to exhibit Markov beliefs, i.e., their decisions depend only on the payoff-relevant state variable (environmental protection $k_{t}$ ) and not on the history of past decisions. Moreover, all agents are symmetric with respect to intertemporal preferences. Thus, agent $t$ 's present value of all future discounted payoffs $W_{t}$ equals 3

$$
W_{t}=\sum_{\tau=0}^{\infty} \delta_{\tau} P\left(i_{t+\tau}, k_{t+\tau}\right),
$$

where $\delta_{\tau}>0$ denotes the discount factor in period $t+\tau$, which is the product of all per-period discount factors $\sigma_{\nu}$ for $\nu \leq \tau$

$$
\delta_{\tau}=\prod_{\nu=0}^{\tau} \sigma_{\nu} .
$$

Following Karp (2007) and Karp and Fuiii (2008) we assume that the per-period discount factors $\sigma_{\nu}$ become constant after a finite time $T$. That is, $\sigma_{\nu}=\beta, \nu>T$, and thus $\delta_{\tau}=\delta_{T} \beta^{\tau-T}, \tau \geq T$. We further concentrate on the case of declining discount rates, which corresponds to (weakly) increasing per-period discount factors, i.e., $\sigma_{\nu} \leq \sigma_{\nu+1}<\beta<1,0<\nu<T$. Without loss of generality, we normalize $\delta_{0}=\sigma_{0}$ to unity. We achieve standard exponential discounting for $T=0$, i.e., $\delta_{\tau}=\beta^{\tau}$. Quasi-hyperbolic discounting (Laibson, 1997, Laibson, 1998, Harris and Laibson, 2001, Salanié and Treich, 2006) corresponds to $T=1$ and $\sigma_{1}=\alpha \beta, 0<\alpha<1$, implying that $\sigma_{1}$ is smaller than all constant subsequent per-period discount factors $\sigma_{\nu}=\beta, \nu>1$.

In the following, we analyze the optimal investment in environmental protection given that agents are committed, naive or sophisticated. In particular, we derive conditions under which all agents never invest in environmental protection although all agents prefer investments in environmental protection in the long run.

anthropogenic greenhouse effect. While the costs occur today, the benefits spread over several decades or even centuries.

${ }^{3}$ We assume that the infinite sum does not diverge. Formally, this requires that in the long run $P$ does not grow faster than the discount factors decline: $\lim _{t \rightarrow \infty} P\left(i_{t}, k_{t}\right) \leq A \beta^{t}$ with some constant $A>0$. 


\section{$3 \quad$ Ex Ante Optimal Investment Plan}

First, we derive the ex ante optimal investment plan. This is the plan agent $t$ achieves by maximizing intertemporal utility, assuming that all future investment decisions will be carried out according to this plan. Note that the ex ante optimization problem is non-stationary if $T \geq 1$. This implies that an investment rule which only depends on the stock of environmental protection, $i_{t}=\phi\left(k_{t}\right)$, does not exist. As a consequence, we cannot derive the ex ante optimal plan via a dynamic programming approach.

The ex ante optimal control problem of agent $t$ is given by:

$$
\max _{\left\{i_{\tau}\right\}_{\tau=t}^{\infty}} \sum_{\tau=0}^{\infty} \delta_{\tau} P\left(i_{t+\tau}, k_{t+\tau}\right)
$$

subject to (11), $i_{\tau} \geq 0, \forall \tau \geq t$ and given $k_{t}$. As we do not assume Inada conditions to hold for the payoff function $P$, we explicitly consider corner solutions, i.e., $i_{\tau}=0, \forall \tau \in[t, \infty)$. Introducing the shadow price $p_{\tau}^{k}$ for the stock of environmental protection and a Kuhn-Tucker variable $p_{\tau}^{i}$ to control for the non-negativity of investment, we obtain the following Lagrangian $\mathcal{L}$ :

$$
\mathcal{L}=\sum_{\tau=0}^{\infty} \delta_{\tau} P\left(i_{t+\tau}, k_{t+\tau}\right)+\sum_{\tau=0}^{\infty} p_{\tau+1}^{k}\left[k_{t+\tau}+i_{t+\tau}-k_{t+1+\tau}\right]+\sum_{\tau=0}^{\infty} p_{\tau}^{i} i_{t+\tau}
$$

Hence, the first order conditions for an optimal intertemporal investment plan read:

$$
\begin{aligned}
& p_{\tau+1}^{k}=-\delta_{\tau} P_{i}\left(i_{t+\tau}, k_{t+\tau}\right)-p_{\tau}^{i} \\
& p_{\tau+1}^{k}=p_{\tau}^{k}-\delta_{\tau} P_{k}\left(i_{t+\tau}, k_{t+\tau}\right) \\
& p_{\tau}^{i} \geq 0, \quad p_{\tau}^{i} i_{t+\tau}=0
\end{aligned}
$$

Because of the strict concavity of the Lagrangian (strictly concave objective function and linear restrictions), these necessary conditions are also sufficient if, in addition, the following transversality condition holds:

$$
\lim _{\tau \rightarrow \infty} p_{\tau}^{k} k_{t+\tau}=0
$$

The strict concavity of the Lagrangian $\mathcal{L}$ also ensures the uniqueness of the optimal investment path $\left\{i_{t+\tau}\right\}_{\tau=0}^{\infty}$.

Equation (6b) is a difference equation, which can be solved unambiguously by taking into account the transversality condition (17):

$$
p_{\tau}^{k}=\sum_{\nu=0}^{\infty} \delta_{\tau+\nu} P_{k}\left(i_{\tau+\nu}, k_{\tau+\nu}\right)
$$

Thus, along the optimal investment path the shadow price of environmental protection equals the present value of the accumulated future utility gains of an additional marginal unit of environmental protection. Inserting the shadow price of environ- 
mental protection (8) into equation (6a), we obtain the following necessary and sufficient condition for an ex ante optimal plan:

$$
-\delta_{\tau} P_{i}\left(i_{t+\tau}, k_{t+\tau}\right)-p_{\tau}^{i}=\sum_{\nu=1}^{\infty} \delta_{\tau+\nu} P_{k}\left(i_{t+\tau+\nu}, k_{t+\tau+\nu}\right), \quad \forall \tau \geq 0 .
$$

Equation (9) states that if positive investment in environmental protection is optimal in period $t+\tau$ (i.e., $p_{\tau}^{i}=0$ ), agent $t$ invests to such an extent that the marginal utility loss at time $t+\tau$ due to the investment in environmental protection (left hand side) equals the present value of the future marginal utility gains of this investment (right hand side). However, if this condition cannot be met for any positive investment $i_{t+\tau}$, then no investment in environmental protection is optimal (i.e., $p_{\tau}^{i} \geq 0$ ). The following proposition characterizes the ex ante optimal investment plan:

\section{Proposition 1 (Ex ante optimal investment plan)}

For the ex ante optimal investment plan of agent t the following statements hold:

1. Optimal investment equals zero for all periods $\tau \geq 0$ if and only if

$$
-\frac{P_{i}\left(0, k_{t}\right)}{P_{k}\left(0, k_{t}\right)} \geq \frac{\beta}{1-\beta}
$$

2. Optimal investment in period $\tau=0$ equals zero if

$$
-\frac{P_{i}\left(0, k_{t}\right)}{P_{k}\left(0, k_{t}\right)} \geq \frac{\beta}{1-\beta}+\frac{1}{1-\beta} \sum_{\nu=1}^{T} \delta_{\nu-1}\left(\sigma_{\nu}-\beta\right) .
$$

3. The unique steady state of environmental protection, $k^{\star}$, is given by

(a) $k^{\star}=k_{t}$ if $i_{t+\tau}=0, \forall \tau \geq 0$,

(b) the unique solution of the implicit equation

$$
\begin{array}{r}
-\frac{P_{i}\left(0, k^{\star}\right)}{P_{k}\left(0, k^{\star}\right)}=\frac{\beta}{1-\beta}, \\
\text { if } i_{t+\tau}>0 \text { for some } \tau \geq 0 .
\end{array}
$$

The proof of Proposition 1 is given in the appendix.

As shown in the appendix, Equations (10)-(12) are derived from the necessary and sufficient condition (9) comparing costs and the discounted sum of benefits of an additional marginal unit of investment. The first part of Proposition 1 says that no investment is optimal from an ex ante point of view only if the costs of the first marginal unit of investment into environmental protection exceed the net present value of benefits at all times $\tau \geq 0$. As $\beta>\sigma_{\tau}$ for $\tau \leq T$, the benefits of the first marginal unit of investment in environmental protection in period $t+\tau$ increase with $\tau$ until $\tau=T$ and stay constant thereafter. Thus, if there is any investment along the ex ante optimal plan, that is condition (10) is violated, then investment in period $t+T$ is strictly positive but investment in former periods may 
be zero. As a consequence, the no-investment condition (10) only depends on the long-run per-period discount factor $\beta$. In fact, comparing the costs and discounted sum of benefits for the first marginal unit of investment at time $t$, the second part gives a sufficient condition for which investment in the first period $\tau=0$ is not optimal. The condition is only sufficient but not necessary, as it may be optimal for agent $t$ not to invest in the first period $\tau=0$ even if condition (10) is violated. This holds, because if there is any investment in environmental protection at all there is at least investment in period $\tau=T$. These investments in later periods decrease the marginal benefits of investments in the first period due to the assumed curvature properties of $P$. The third part of Proposition 1 establishes the existence of a unique steady state of environmental protection $k^{\star}$ of the ex ante optimal plan, which is equal to the initial stock $k_{t}$ if no investment is optimal. Otherwise, it is implicitly given by equation (12), which balances the long-run costs and benefits of an additional marginal unit of investment. Uniqueness follows from the assumed curvature properties of $P$.

The first and second part of Proposition 1 lalso imply that it may be optimal from an ex ante perspective to invest in environmental protection in the long run but not in the short run. In the following, we will say agent $t$ postpones investment if $i_{t}=0$ but $i_{t+\tau}>0$ for some $\tau>0$. The following corollary gives sufficient conditions for postponing investment to be ex ante optimal.

\section{Corollary 1}

It is ex ante optimal for agent $t$ to postpone investment in environmental protection if

$$
\frac{\beta}{1-\beta}>-\frac{P_{i}\left(0, k_{t}\right)}{P_{k}\left(0, k_{t}\right)} \geq \frac{\beta}{1-\beta}+\frac{1}{1-\beta} \sum_{\nu=1}^{T} \delta_{\nu-1}\left(\sigma_{\nu}-\beta\right) .
$$

The proof of Corollary 1 follows directly from Proposition 1 .

Corollary 1 says that postponing investment is ex ante optimal if condition (11) holds but condition (10) is violated. A main insight of Corollary 1 is that postponing investment can be ex ante optimal only for hyperbolically discounting agents. If agents discount exponentially (i.e., $T=0$ ), the last term of the RHS of condition (13) vanishes, and thus the condition cannot hold 4

\section{Ex Post Implemented Investment}

For $T \geq 1$ the ex ante optimal plan of agent $t$ is not ex ante optimal for future agents if investment is ex ante optimal, i.e., condition (10) is violated, because of the non-stationarity of agents' preferences. Therefore, future agents may not stick to the ex ante optimal plan of their predecessors. As a consequence, the investment that is actually implemented ex post may differ from the ex ante optimal plan,

\footnotetext{
${ }^{4}$ Note, however, that postponing investment can also be optimal for exponentially discounting agents within other model frameworks. In particular, this is the case if future outcomes are risky (see, for example, the option value framework developed by Dixit, 1992 and Dixit and Pindvck, 1994).
} 
depending on the behavioral pattern assumed. In the following, we analyze the ex post implemented investment under commitment and for naive and sophisticated agents. We show that, if condition (13) holds, i.e., investment is ex ante optimal in the long run but not in the short run, only committed agents will invest in environmental protection.

\subsection{Commitment}

If the first agent, agent zero, has the power to enforce her ex ante optimal plan, she will certainly do so, as this is, by definition, the unique plan which maximizes her intertemporal utility. According to the analysis of the former section, investment in environmental protection is ex ante optimal, if condition (10) is violated. Obviously, in the case of such a commitment, the ex post implemented plan is equal to the ex ante optimal plan. The following proposition summarizes this insight.

\section{Proposition 2 (Commitment)}

If all subsequent agents are committed to the ex ante optimal plan of agent zero, the following statements hold:

1. There is no investment in environmental protection if and only if

$$
-\frac{P_{i}\left(0, k_{0}\right)}{P_{k}\left(0, k_{0}\right)} \geq \frac{\beta}{1-\beta}
$$

2. The unique steady state of environmental protection, $k^{\star}$, is given by

(a) $k^{\star}=k_{0}$ if $i_{t}=0, \forall t \geq 0$,

(b) the unique solution of the implicit equation

$$
\begin{gathered}
-\frac{P_{i}\left(0, k^{\star}\right)}{P_{k}\left(0, k^{\star}\right)}=\frac{\beta}{1-\beta}, \\
\text { if } i_{t}>0 \text { for some } t \geq 0 .
\end{gathered}
$$

The proof of Proposition 2 follows directly from Proposition 1 ,

If no commitment mechanism is available, the ex post outcome depends on the agents' awareness of the time-inconsistency problem. Following the standard approach, we distinguish two different behavioral patterns.

\subsection{Naive Agents}

Agents are naive, if they are not aware that the ex ante optimal plans of subsequent agents may differ from their own ex ante optimal plan. As a consequence, the naive agent invests in environmental protection if and only if it is ex ante optimal to invest in the first period. The following proposition elaborates on the ex post implemented investment in case of naive agents. 


\section{Proposition 3 (Naive agents)}

If agents are naive and cannot be bound to the ex ante optimal plan of agent zero, the following statements hold:

1. Agent $t$ invests in environmental protection if and only if it is ex ante optimal to invest in period $t$.

2. A sufficient condition for optimal ex ante investment in period t to equal zero is given by condition (11).

3. For the unique steady state of environmental protection, $k^{n}$, the following conditions hold:

(a) $k^{n}=k_{0}$ if $i_{t}=0, \forall t \geq 0$,

(b) $k^{n}<\bar{k}^{n}$ where $\bar{k}^{n}$ is given by the solution of the implicit equation

$$
\begin{aligned}
& \qquad \frac{P_{i}\left(0, \bar{k}^{n}\right)}{P_{k}\left(0, \bar{k}^{n}\right)}=\frac{\beta}{1-\beta}+\frac{1}{1-\beta} \sum_{\nu=1}^{T} \delta_{\nu-1}\left(\sigma_{\nu}-\beta\right), \\
& \text { if } i_{t}>0 \text { for some } t \geq 0 .
\end{aligned}
$$

Proof: Ad 1. By definition naive agents believe that all succeeding agents stick to their ex ante optimal plan. Given this believe, it is optimal for agent $t$ to follow her ex ante optimal plan. As a consequence, agent $t$ only invests in period $t$ if this is ex ante optimal.

Ad 2. We know from Proposition 1 that condition (11) is a sufficient condition for no investment to be ex ante optimal.

Ad 3. The case of no investment is trivial. Suppose investment is positive for some $t \geq 0$. Any level of environmental protection $k$ can only be a steady state if all subsequent agents do not invest, for which a sufficient condition is condition (11). Denoting $\bar{k}^{n}$ as the stock of environmental protection for which this condition holds with equality, the steady state stock $k^{n}$ is strictly below this level, as condition (11) is just sufficient but not necessary. Again, the steady state is unique due to the curvature properties of $P$ (see proof of part three of Proposition 1).

Proposition 3 says that agent $t$ only invests in environmental protection in period $t$ if the ex ante optimal plan suggests to do so. It also implies that naive agents never invest in environmental protection if agent zero does not invest in environmental protection, because then all subsequent agents' ex ante optimal plans are identical to the ex ante optimal plan of agent zero, as $k_{t+1}=k_{t}$ if $i_{t}=0$. In particular, this holds if condition (13) holds. In this case it is ex ante optimal for agent zero not to invest herself but for future agents to invest. As naive agents do not forsee that future agents do not stick to their ex ante optimal plan, there is no investment at all in environmental protection over the infinite time horizon although all agents prefer investment in the long run. 


\subsection{Sophisticated Agents}

In contrast to naive agents, sophisticated agents anticipate future agents' deviations from their ex ante optimal plan. As agents can only influence future agents' decisions by influencing the stock of environmental protection, time consistent planning of all agents can be viewed as playing a non-cooperative sequential game. We seek symmetric Markov perfect equilibria, i.e., stationary investment rules only depending on the stock of environmental protection, $i_{t}=\phi\left(k_{t}\right)$, which are mutually best responses for all agents. Thus, an equilibrium investment rule satisfies

$$
\phi\left(k_{t}\right)=\arg \max _{i_{t}}\left[P\left(i_{t}, k_{t}\right)+\sum_{\tau=1}^{\infty} P\left(\phi\left(k_{t+\tau}\right), k_{t+\tau}\right)\right],
$$

subject to equation (11) and $\phi\left(k_{t}\right) \geq 0, \forall t \geq 0$.

In order to apply a dynamic programming approach we rewrite the intertemporal utility (2) of agent $t$ to yield (Karp and Fuiii, 2008):

$$
\begin{aligned}
W_{t} & =P\left(i_{t}, k_{t}\right)+\sum_{\tau=1}^{\infty} \delta_{\tau} P\left(i_{t+\tau}, k_{t+\tau}\right) \\
& =P\left(i_{t}, k_{t}\right)+\sum_{\tau=1}^{T} \delta_{\tau} P\left(i_{t+\tau}, k_{t+\tau}\right)+\sum_{\tau=T+1}^{\infty} \beta \delta_{\tau-1} P\left(i_{t+\tau}, k_{t+\tau}\right) \\
& =P\left(i_{t}, k_{t}\right)+\sum_{\tau=1}^{T}\left(\delta_{\tau}-\beta \delta_{\tau-1}\right) P\left(i_{t+\tau}, k_{t+\tau}\right)+\beta \sum_{\tau=0}^{\infty} \delta_{\tau} P\left(i_{t+1+\tau}, k_{t+1+\tau}\right)
\end{aligned}
$$

Then, we can write agent $t$ 's optimization problem recursively by introducing the value function $V$ :

$$
V\left(k_{t}\right)=\max _{i_{t}}\left[P\left(i_{t}, k_{t}\right)+\sum_{\tau=1}^{T}\left(\delta_{\tau}-\beta \delta_{\tau-1}\right) P\left(\phi\left(k_{t+\tau}\right), k_{t+\tau}\right)+\beta V\left(k_{t+1}\right)\right] .
$$

Assuming a differentiable equilibrium investment rule, we obtain the following Euler equation (as shown in the appendix):

$$
\begin{aligned}
-P_{i}\left(\phi\left(k_{t}\right), k_{t}\right) \geq & \sum_{\tau=1}^{T}\left(\delta_{\tau}-\beta \delta_{\tau-1}\right)\left[P_{i}\left(\phi\left(k_{t+\tau}\right), k_{t+\tau}\right) \phi^{\prime}\left(k_{t+\tau}\right)+P_{k}\left(\phi\left(k_{t+\tau}\right), k_{t+\tau}\right)\right] \\
& +\beta\left[P_{k}\left(\phi\left(k_{t+1}\right), k_{t+1}\right)-P_{i}\left(\phi\left(k_{t+1}\right), k_{t+1}\right)\right]
\end{aligned}
$$

where the inequality sign corresponds to the corner solution $\phi\left(k_{0}\right)=0$. In general, the equilibrium investment rules and the corresponding steady states are not unique (see Karp, 2005, Karp, 2007 and Karp and Fuiii, 2008). The reason is that the equation of motion for the stock of environmental protection (10) and the Euler equation (20) constitute an underdetermined system of equations for the unknowns $k_{t}, \phi\left(k_{t}\right)$ and $\phi^{\prime}\left(k_{t}\right)$. However, the following proposition establishes that under certain conditions there exists a unique equilibrium in which all agents do not invest. 


\section{Proposition 4 (Sophisticated agents)}

If agents are sophisticated and cannot be bound to the ex ante optimal plan of agent zero, the following statements hold:

1. No investment in all periods $t \geq 0$ is the unique equilibrium if and only if

$$
-\frac{P_{i}\left(0, k_{0}\right)}{P_{k}\left(0, k_{0}\right)} \geq \frac{\beta}{1-\beta}+\frac{1}{1-\beta} \sum_{\nu=1}^{T} \delta_{\nu-1}\left(\sigma_{\nu}-\beta\right) .
$$

2. For the set of asymptotically stable steady state(s) of environmental protection, $k^{s}$, the following condition holds:

(a) $k^{s}=k_{0}$ if condition (21) holds.

(b) $k^{s} \in\left[\max \left(\underline{k}^{s}, k_{0}\right), \bar{k}^{s}\right]$ where $\underline{k}^{s}$ and $\bar{k}^{s}$ are given by the solutions of the implicit equations

$$
\begin{aligned}
& -\frac{P_{i}\left(0, \underline{k}^{s}\right)}{P_{k}\left(0, \underline{k}^{s}\right)}=\frac{\beta}{1-\beta}+\frac{1}{1-\beta}\left(1-\frac{P_{i}\left(0, \underline{k}^{s}\right)}{P_{k}\left(0, \underline{k}^{s}\right)}\right) \sum_{\nu=1}^{T} \delta_{\nu-1}\left(\sigma_{\nu}-\beta\right), \\
& -\frac{P_{i}\left(0, \bar{k}^{s}\right)}{P_{k}\left(0, \bar{k}^{s}\right)}=\frac{\beta}{1-\beta}+\frac{1}{1-\beta} \sum_{\nu=1}^{T} \delta_{\nu-1}\left(\sigma_{\nu}-\beta\right),
\end{aligned}
$$

if if condition (21) is violated.

The proof of Proposition 4 is given in the appendix.

The first part of Proposition 4 says that no agent will invest in environmental protection if condition (21) holds, which trades off the costs and present value of all future benefits of the first marginal unit of investment. Condition (21) is identical to condition (11), the sufficient condition for naive agents to never invest in environmental protection. However, for sophisticated agents the condition is not only sufficient but also necessary. The reason is that sophisticated agents anticpate future agents' deviations from their ex ante optimal plan. As a consequence, they have stronger incentives to invest compared to naive agents who postpone investments due to the ill-founded believe that later agents will invest. Note that if condition (21) holds the equilibrium is only unique with respect to the outcome $i_{t}=0, k_{t}=k_{0}, \forall t \geq 0$ but not with respect to the underlying equilibrium investment rule. In fact, all investment rules $\phi\left(k_{t}\right)$ with $\phi\left(k_{0}\right)=0$ and $-1 \leq \phi^{\prime}\left(k_{0}\right) \leq 0$ are equilibrium investment rules.

An asymptotically stable steady state implies for the equilibrium investment rule $-1 \leq \phi^{\prime}\left(k^{s}\right) \leq 0$. However, unlike the case of exponential discounting, the restriction to asymptotically stable steady states in combination with Markov beliefs does not yield a unique steady state in equilibrium. In fact, there exists a whole interval of possible equilibrium steady states, whose lower bound $\underline{k}^{s}$ corresponds to the equilibrium investment rule with $\phi^{\prime}\left(k^{s}\right)=-1$ and whose upper bound $\bar{k}^{s}$ corresponds to the equilibrium investment rule with $\phi^{\prime}\left(k^{s}\right)=0$. If $k_{0} \geq \underline{k}^{s}$ then no 
investment in all periods is an equilibrium steady state, although (21) is violated, but it is not the unique equilibrium.

As $\phi^{\prime}\left(k^{s}\right) \leq 0$, investments in environmental protection of subsequent agents are strategic substitutes at least in a neighborhood around an asymptotically stable steady state. That is, the more agent $t$ invests the less agent $t+1$ will invest. It seems intuitive to think of investment decisions of subsequent agents as strategic substitutes, as the marginal benefits of investments are non-decreasing in the stock of environmental protection (i.e., $P_{i k} \leq 0$ ). However, this intuition only holds for a neighborhood around the equilibrium steady state and may be violated along the equilibrium trajectory.

\subsection{Comparison}

Using Propositions 2 24, we now compare the ex post outcomes of committed, naive and sophisticated agents. First, we compare the investment decisions between the three behavioral regimes.

\section{Corollary 2 (Investment comparison: now or never)}

For both naive and sophisticated agents the following statement holds:

$$
i_{0}=0 \quad \Rightarrow \quad i_{t}=0, \quad \forall t>0
$$

The proof of Corollary 2 follows directly from Propositions 3 and 4.

Corollary 2 says neither naive nor sophisticated agents postpone investment in environmental protection, i.e., no investment in period $t=0$ but positive investment in some later period $t>0$. The reason is straightforward. Naive agents always stick to their ex ante optimal plan. If investment is not optimal in period $t$ the ex ante optimal plan of agent $t+1$ is identical to the ex ante optimal plan of agent $t$, as $k_{t+1}=$ $k_{t}$. Sophisticated agents invest according to an investment rule $\phi\left(k_{t}\right)$. If $\phi\left(k_{t}\right)=0$ then $\phi\left(k_{t+1}\right)=0$, as $k_{t+1}=k_{t}$. Thus, postponing investment can only occur if agent zero can commit future agents to her ex ante optimal plan. In particular, if condition (13) of Corollary 1 holds, there will be no investment in environmental protection in all periods, no matter whether agents are sophisticated or naive, although all agents would prefer investment in the long run. Thus, awareness of the time-inconsistency of own preferences, i.e., "switching" from a naive to a sophisticated agent, does not necessarily improve outcomes.

This does not imply, however, that investment decisions of naive and sophisticated agents are identical. On the contrary, if, for example, condition (21) is just violated 5 then there exist an equilibrium investment rule with $\phi\left(k_{0}\right)>0$ while it is still not ex ante optimal to invest in $t=0$ and, therefore, naive agents will never invest. The intuition is that the benefits of investments today decrease with investments in future periods. If condition (21) is just violated this also holds for condition (14). Accordingly, it is ex ante optimal for agent zero to invest at least in the long run, which implies $i_{T}>0$. Thus, as naive agents wrongly believe that agents in the future will invest according to their ex ante optimal plan, investment seems less

\footnotetext{
${ }^{5}$ More formally: $-\frac{P_{i}\left(0, k_{0}\right)}{P_{k}\left(0, k_{0}\right)}=\epsilon+\frac{\beta}{1-\beta}+\frac{1}{1-\beta} \sum_{\nu=1}^{T} \delta_{\nu-1}\left(\sigma_{\nu}-\beta\right)$ for some sufficiently small $\epsilon>0$.
} 
beneficial for them compared to sophisticated agents who correctly anticipate future agents' deviations from their ex ante optimal plan.

Second, the following corollary compares the steady states for the different behavioral regimes.

\section{Corollary 3 (Steady state comparison)}

For the steady states of committed, naive and sophisticates agents the following relationships hold:

1. $k^{\star}=\bar{k}^{s}=k^{n}=k_{0}$ if condition (14) holds,

2. $k^{\star}>\bar{k}^{s}=k^{n}=k_{0}$ if condition (13) holds,

3. and $k^{\star}>\bar{k}^{s}>k^{n} \geq k_{0}$ if condition (21) is violated.

The proof of Corollary 3 follows directly from Propositions 2 , 4.

The first part of Corollary 3 captures the case that it is ex ante optimal to never investment in environmental protection. Then, also naive and sophisticated agents will never invest. The second part says that if condition (13) holds, it is ex ante optimal to postpone investment, but there will be no investment in case of naive or sophisticated agents. The third part refers to the case for which investment is ex ante optimal and also sophisticated agents invest. As already outlined, this does not necessarily imply that naive agents also invest. Moreover, the highest achievable equilibrium steady state for sophisticated agents $\bar{k}^{s}$ exceeds the steady state in case of naive agents $k^{n}$. Thus, in this case awareness of the time-inconsistency problem may overcome the no investment outcome of naive agents if sophisticated agents can coordinate on the equilibrium investment rule leading to $\bar{k}^{s}$. Nevertheless, even the highest equilibrium steady state in case of sophisticated agents falls short of the ex ante optimal steady state level of environmental protection. Thus, in the long run both naive and sophisticated agents are stuck in a situation in which it is ex ante optimal for them and all subsequent agents to further invest in environmental protection, but no generation will do so. This is not only unsatisfactory for all agents, but may also be Pareto inefficient, as the following proposition states.

\section{Proposition 5 (Efficiency)}

For the intertemporal utility of committed, naive and sophisticated agents the following statements hold:

1. Enforcement of the ex ante optimal plan is always Pareto optimal.

2. The ex post implemented investment plans of naive and sophisticated agents may be inefficient in the sense that there exist Pareto superior investment plans.

The proof of Proposition 5 is given in the appendix.

Obviously the ex ante optimal plan of agent $t$ is Pareto optimal as any deviation from it decreases intertemporal utility of agent $t$. It is not surprising that the ex post implemented plans of naive and sophisticated agents may be inefficient (although naive agents are not aware of this fact as they always assume their ex ante optimal plan will be carried out), it is rather surprising that they may not 
be inefficient. The reason is that utility cannot be directly transferred between different agents. The only way of utility transfers in this model is via investments in environmental protection. As environmental protection is assumed to be bounded, it is not possible to compensate the utility loss of one agent due to an additional investment by ever increasing investments of future agents (i.e., Ponzi games are ruled out). However, whether a decreasing series of additional investments can constitute a Pareto improvement among all agents depends on the series of perperiod discount factors.

\section{Discussion}

In a simple model of environmental protection we have shown that neither naive nor sophisticated agents postpone investments even if this is ex ante optimal. Thus, if agent zero does not invest so do all subsequent agents, although all agents prefer investments in the long run. Of course, not all environmental problems exhibit the property that postponing investments is ex ante optimal. But even if naive or sophisticated agents invest in the first period, society will reach a long-run steady state in which further investments are ex ante optimal but neither naive nor sophisticated agents will invest. In addition, such an outcome may be Pareto inefficient. In the following we discuss some of our model assumptions and hint at immediate policy implications.

We assumed that the stock of environmental protection does not depreciate. Note that due to continuity all our results also hold for sufficiently small rates of depreciation. However, if depreciation is sufficiently large, condition (21) is not sufficient anymore for the no investment equilibrium to be unique for sophisticated agents. The reason is that for positive depreciation investment decisions of subsequent agents are not necessarily strategic complements in a neighborhood of the steady state, as asymptotic stability requires that $\phi^{\prime}\left(k^{s}\right) \leq \gamma$, where $\gamma>0$ denotes the constant rate of depreciation of the stock of environmental protection. As a consequence, the RHS of a corresponding sufficient condition for no investment to be the unique equilibrium would increase. Then, depending on the depreciation rate $\gamma$, we might not find a sufficient condition such as condition (13) for which it is ex ante optimal to postpone investment and neither naive nor sophisticated agents invest. However, the core results that neither sophisticated nor naive agents postpone investment, and no commitment to the ex ante optimal plan results in a steady state in which both naive and sophisticated agents would prefer further investments in the long run but no investments are carried out, remain untouched.

The payoff function $P$ was assumed to be time-invariant. This neglects problems where doing nothing worsens the environmental problem such that marginal benefits of investments in environmental protection increase over time. Further, it does not capture technological progress which decreases marginal costs of investment over time. Both extensions may lead to investment of future agents although agent zero did not invest and agent zero cannot commit future agents to her ex ante optimal plan. However, even if naive or sophisticated agents do invest in later periods they invest later and less than would be ex ante optimal, again leading to a steady state where further investments would be ex ante optimal for all subsequent agents but 
are not carried out.

As our results are qualitatively robust, this gives rise to severe concern for the performance of long run environmental policy if decision makers exhibit timeinconsistent preferences and cannot easily commit themselves and future decision makers to an ex ante optimal plan. First, we want to emphasize that the weak policy performance with respect to some long-run environmental problems is consistent with the assumption of declining discount rates of decision makers. As an example think of the problem of nuclear waste disposal 6 It was obvious from the very beginning of the civilian utilization of nuclear fission for energy generation in the 1950s that there will be non-recycable wastes that are highly radioactive for up to ten thousands of years. Yet, the solution to the disposal problem has been continually postponed and still no long-run storage site for radioactive wastes exists. Moreover, it was not until the 1970s that nuclear waste disposal became a source of concern and governments commissioned research in this area.

Another example is the slow progress in stabilizing the emissions of greenhouse gases to prevent, or at least reduce, anthropogenic climate change 7 The Framework Convention on Climate Change, which was open to signature in Rio de Janeiro in June 1992 and at the UN headquarters thereafter, received the signatures of 186 states. The signatory developed countries agreed as a first step to stabilize their greenhouse gas emissions at their 1990 levels by 2000. Most countries failed to do so. Similar outcomes can be observed with the subsequent Kyoto protocol which was signed in December 1997. In this treaty the developed countries agreed to reduce their greenhouse gas emissions to $95 \%$ of their 1990 levels by 2008-2012. Some countries which signed the protocol later refused to ratify it (e.g., USA and Australia). Also many countries which ratified Kyoto are still far from their promised emission targets. Moreover, the countries which already met (or are likely to meet) their targets have done so more by accident than by deliberate action (Pearce, $2003)$. Both examples fit well with the behavior we would expect if governments make decisions on the basis of hyperbolic discounting (although they might be not aware of it) and the ex ante optimal plan suggests to postpone investment to later periods. Although governments might have intended to act in the future they fail to do so because of the time-inconsistency of their preferences 8

Our analysis suggests that, although under certain conditions sophisticated agents perform as badly as naive agents, awareness of the time-inconsistency problem may be a short run remedy for the no investment outcome. As argued, sophisticated agents may invest in environmental protection although it is ex ante optimal to postpone investment and, thus, naive agents would never invest. However, sophisticated agents would have to coordinate on the "right" equilibrium and even the

\footnotetext{
${ }^{6}$ For a more detailed exposition of the nuclear fuel cycle see, for example, Proops (2001) and Wilson (1996).

${ }^{7}$ Although anthropogenic climate change is a stock pollutant problem which is likely to exhibit increasing marginal damage, the case is applicable to the results of the model presented as the Framework Convention on Climate Change and the Kyoto protocol only limit emissions.

${ }^{8}$ Of course, we do not claim that hyperbolic discounting is the only possible explanation for the observed underperformance with respect to nuclear waste disposal and mitigating climate change. In fact, political economy issues, public good characteristics and externalities also play a crucial role.
} 
maximal reachable steady state falls short of the ex ante optimal levels of environmental protection. As a consequence, also sophisticated agents are eventually stuck in a situation where further investment in the long run is ex ante optimal for all subsequent agents but no one will invest.

\section{Conclusion}

We have analyzed optimal intertemporal investments in environmental protection for a society consisting of hyperbolically discounting agents. Because of the nonstationarity of hyperbolic preferences the ex post observed outcome crucially depends on additional behavioral constraints. As prime examples we have discussed the committed, the naive and the sophisticated agents. Within our model framework neither naive nor sophisticated agents postpone investment even if this is ex ante optimal. Thus, if agent zero does not invest so do all subsequent agents, although all agents prefer investments in the long run. Such an outcome is not only unsatisfactory for each generation, it may also be Pareto inefficient.

Our results give rise to concern, as they are consistent with real world observations of weak policy performance with respect to long-run environmental problems. Moreover, underperformance is not just a consequence of naivety, i.e., the missing awareness of the time-inconsistency problem. Although awareness of the timeinconsistency problem may act as short-run remedy, the resulting long-run steady states of environmental protection are below the ex ante optimal levels. Thus, if decision makers exhibit hyperbolic preferences, we have to do more than taking time-inconsistency seriously. We also have to overcome the (potential) inefficiency of time-consistent equilibria.

Obviously, a commitment device would help. Cropper and Laibson (1999), for example, suggest to Pareto improve the outcome by subsidizing the interest rate. Their crucial assumption is that the effect of implemented policies occur with a timelag, which is equivalent to a commitment mechanism for the next period. However, they only consider quasi-hyperbolic discounting implying that only a commitment device for one period is necessary. In the general setting of our model, commitment for $T$ periods would be needed. However, in a long-term intergenerational setting, the enforcement power of the present generation is limited (and also questionable on ethical grounds, as this implies a dictatorship of the present over the future generations). Hence, the solution of this problem is open to future research.

Finally, it is worth noting that, although our model was primarily designed to address long-run environmental problems, the results extend to other investment decisions of a long-run and intergenerational nature, such as education, health insurance and pension schemes. 


\section{Appendix}

\section{A.1 Proof of Proposition 1}

Ad 1. Assume that it is optimal not to invest in all periods, i.e., $i_{t+\tau}=0, \forall \tau \geq 0$. Then, it follows from (II) that $k_{t+\tau}=k_{t}, \forall \tau \geq 0$. Inserting into the necessary and sufficient condition (9) and recalling that the Kuhn-Tucker parameter $p_{\tau}^{i} \geq 0$ if $i_{t+\tau}=0$ yields

$$
-\frac{P_{i}\left(0, k_{t}\right)}{P_{k}\left(0, k_{t}\right)} \geq \sum_{\nu=1}^{\infty} \frac{\delta_{\tau+\nu}}{\delta_{\tau}}=\left\{\begin{array}{ll}
\sum_{\nu=\tau+1}^{T} \frac{\delta_{\nu}}{\delta_{\tau}}+\frac{\delta_{T}}{\delta_{\tau}} \sum_{\nu=T+1}^{\infty} \beta^{\nu-T}, & \tau<T \\
\sum_{\nu=1}^{\infty} \beta^{\nu}=\frac{\beta}{1-\beta}, & \tau \geq T
\end{array}\right\} .
$$

This condition has to hold for all $\tau$ for the no-investment path $i_{t+\tau}=0, \forall \tau \geq 0$ to be optimal from an ex ante point of view. Note that the expression for $\tau \geq T$ is larger than the expression for $\tau<T$ as $\sigma_{\tau}<\beta, \forall \tau \leq T$. Thus, the inequality holds for all $\tau \in[0, \infty)$ if it holds for $\tau \geq T$.

Ad 2. By assumption $P_{i k}<0$ and $P_{k k}<0$ hold, thus

$$
P_{k}\left(0, k_{t}\right) \geq P_{k}\left(i_{t+\tau}, k_{t+\tau}\right), \quad \forall \tau \geq 0 .
$$

Inserting into the necessary and sufficient condition (9) for $\tau=0$, we obtain the following condition for $i_{t}=0$ to be optimal

$$
\begin{aligned}
P_{i}\left(0, k_{t}\right) & \geq \sum_{\nu=1}^{\infty} \delta_{\nu} P_{k}\left(i_{t+\nu}, k_{t+\nu}\right) \geq P_{k}\left(0, k_{t}\right) \sum_{\nu=1}^{\infty} \delta_{\nu} \\
& =P_{k}\left(0, k_{t}\right)\left[\sum_{\nu=1}^{T} \delta_{\nu}+\sum_{\nu=T+1}^{\infty} \delta_{\nu}\right]=P_{k}\left(0, k_{t}\right)\left[\sum_{\nu=1}^{T} \delta_{\nu}+\delta_{T} \frac{\beta}{1-\beta}\right] \\
& =P_{k}\left(0, k_{t}\right)\left[\frac{\beta}{1-\beta}+\frac{1}{1-\beta} \sum_{\nu=1}^{T} \delta_{\nu}(1-\beta)+\frac{\delta_{T} \beta}{1-\beta}-\frac{\beta}{1-\beta}\right] \\
& =P_{k}\left(0, k_{t}\right)\left[\frac{\beta}{1-\beta}+\frac{1}{1-\beta} \sum_{\nu=1}^{T} \delta_{\nu-1}\left(\sigma_{\nu}-\beta\right)\right] .
\end{aligned}
$$

In the first line, the first inequality stems from neglecting the Kuhn-Tucker parameter $p_{\tau}^{i} \geq 0$ in the necessary and sufficient condition (91), the second inequality sign holds due to condition (A.2). Note that the term in brackets is smaller than $\beta /(1-\beta)$ because the sum is negative as $\beta>\sigma_{\tau}, \forall \tau \geq T$.

Ad 3. The case of no investment is trivial. If investment is ex ante optimal, there exists a $\tau \geq T$ with $i_{t+\tau}>0$. Thus, the steady state cannot be reached before period $t+\tau$. As there is no depreciation, investment has to equal zero in the steady state. Thus, the steady state stock of environmental protection is given by the solution of equation (12). This solution is unique because, due to the assumed curvature properties of $P$, we obtain:

$$
\frac{\partial}{\partial k}\left(-\frac{P_{i}(0, k)}{P_{k}(0, k)}\right)=\frac{P_{k k}(0, k) P_{i}(0, k)-P_{i k}(0, k) P_{k}(0, k)}{P_{k}(0, k)^{2}}>0 .
$$


Thus, the steady state is higher the larger is the long-run per-period discount factor $\beta$.

\section{A.2 Derivation of the Euler equation}

Using the value function (19) the optimization problem of agent $t$ reads

$$
\max _{i_{t}}\left[P\left(i_{t}, k_{t}\right)+\sum_{\tau=1}^{T}\left(\delta_{\tau}-\beta \delta_{\tau-1}\right) P\left(i_{t+\tau}, k_{t+\tau}\right)+\beta V\left(k_{t+1}\right)\right]
$$

subject to equation (11) and $0 \geq i_{\tau}=\phi\left(k_{\tau}\right), \forall \tau \geq t$.

First, note that the following conditions hold:

$$
\frac{\partial k_{t+\tau}}{\partial k_{t}}=\frac{\partial k_{t+\tau}}{\partial k_{t+\tau-1}} \cdots \frac{\partial k_{t+1}}{\partial k_{t}}=1, \quad \frac{\partial k_{t+\tau}}{\partial i_{t}}=\frac{\partial k_{t+\tau}}{\partial k_{t+1}} \frac{\partial k_{t+1}}{\partial i_{t}}=1, \quad \forall \tau>t .
$$

Then, we obtain for the first-order condition

$$
\begin{aligned}
-P_{i}\left(\phi\left(k_{t}\right), k_{t}\right)= & \sum_{\tau=1}^{T}\left(\delta_{\tau}-\beta \delta_{\tau-1}\right)\left[P_{i}\left(\phi\left(k_{t+\tau}\right), k_{t+\tau}\right) \phi^{\prime}\left(k_{t+\tau}\right)\right. \\
& \left.+P_{k}\left(\phi\left(k_{t+\tau}\right), k_{t+\tau}\right)\right]+\beta V^{\prime}\left(k_{t+1}\right) .
\end{aligned}
$$

By the envelope theorem

$$
\begin{aligned}
V^{\prime}\left(k_{t}\right)= & P_{k}\left(\phi\left(k_{t}\right), k_{t}\right)+\sum_{\tau=1}^{T}\left(\delta_{\tau}-\beta \delta_{\tau-1}\right)\left[P_{i}\left(\phi\left(k_{t+\tau}\right), k_{t+\tau}\right) \phi^{\prime}\left(k_{t+\tau}\right)\right. \\
& \left.+P_{k}\left(\phi\left(k_{t+\tau}\right), k_{t+\tau}\right)\right]+\beta V^{\prime}\left(k_{t+1}\right) .
\end{aligned}
$$

Inserting the first-order condition into $V^{\prime}\left(k_{t}\right)$ yields

$$
V^{\prime}\left(k_{t}\right)=P_{k}\left(\phi\left(k_{t}\right), k_{t}\right)-P_{i}\left(\phi\left(k_{t}\right), k_{t}\right) .
$$

Inserting back into the first-order condition, we obtain the Euler equation

$$
\begin{aligned}
-P_{i}\left(\phi\left(k_{t}\right), k_{t}\right)= & \sum_{\tau=1}^{T}\left(\delta_{\tau}-\beta \delta_{\tau-1}\right)\left[P_{i}\left(\phi\left(k_{t+\tau}\right), k_{t+\tau}\right) \phi^{\prime}\left(k_{t+\tau}\right)+P_{k}\left(\phi\left(k_{t+\tau}\right), k_{t+\tau}\right)\right] \\
& +\beta\left[P_{k}\left(\phi\left(k_{t+1}\right), k_{t+1}\right)-P_{i}\left(\phi\left(k_{t+1}\right), k_{t+1}\right)\right] .
\end{aligned}
$$

However, it may be that the Euler equation does not hold for non-negative investments $i_{t}=\phi\left(k_{t}\right)$. In this case, the optimal investment is $i_{t}=\phi\left(k_{0}\right)=0, \forall t \geq 0$ and the following inequality holds

$$
\begin{aligned}
-P_{i}\left(0, k_{0} t\right) \geq & \sum_{\tau=1}^{T}\left(\delta_{\tau}-\beta \delta_{\tau-1}\right)\left[P_{i}\left(0, k_{0}\right) \phi^{\prime}\left(k_{0}\right)+P_{k}\left(0, k_{0}\right)\right] \\
& +\beta\left[P_{k}\left(0, k_{0}\right)-P_{i}\left(0, k_{0}\right)\right] .
\end{aligned}
$$

\section{A.3 Proof of Proposition 4}

Ad 1. First, note that exploding equilibria with an ever increasing stock of environmental protection are ruled out by the boundedness of $k \in[0, \bar{k}]$. As a consequence, the stock of 
environmental protection converges to a steady state in equilibrium. Asymptotic stability of the steady state implies $-1 \leq \phi^{\prime}\left(k^{s}\right) \leq 0$. Now, we show that if no investment is the unique equilibrium then condition (21) holds and vice versa.

" $\Rightarrow$ ": Suppose, no investment in all periods is the unique equilibrium. Inserting into the Euler equation and re-arranging terms yields

$$
\begin{aligned}
-\frac{P_{i}\left(0, k_{0}\right)}{P_{k}\left(0, k_{0}\right)} & \geq \frac{\beta}{1-\beta}+\frac{1}{1-\beta}\left(1+\frac{P_{i}\left(0, k_{0}\right) \phi^{\prime}\left(k_{0}\right)}{P_{k}\left(0, k_{0}\right)}\right) \sum_{\nu=1}^{T} \delta_{\nu-1}\left(\sigma_{\nu}-\beta\right) \\
& \geq \frac{\beta}{1-\beta}+\frac{1}{1-\beta} \sum_{\nu=1}^{T} \delta_{\nu-1}\left(\sigma_{\nu}-\beta\right) .
\end{aligned}
$$

The inequality sign in the second line holds because $P_{i}<0, P_{k}>0, \phi^{\prime} \leq 0$ and $\sum_{\nu=1}^{T} \delta_{\nu-1}\left(\sigma_{\nu}-\beta\right)<0$, as $\sigma_{\nu}<\beta, \forall \nu \geq T$.

" $\Leftarrow$ ": Suppose that condition (21) holds. Suppose further that there exists an asymptotically stable steady state with $k^{s}>k_{0}$. Inserting into the Euler equation and rearranging terms yields:

$$
\begin{aligned}
-\frac{P_{i}\left(0, k^{s}\right)}{P_{k}\left(0, k^{s}\right)} & =\frac{\beta}{1-\beta}+\frac{1}{1-\beta}\left(1+\frac{P_{i}\left(0, k^{s}\right) \phi^{\prime}\left(k^{s}\right)}{P_{k}\left(0, k^{s}\right)}\right) \sum_{\nu=1}^{T} \delta_{\nu-1}\left(\sigma_{\nu}-\beta\right) \\
& \leq-\frac{P_{i}\left(0, k_{0}\right)}{P_{k}\left(0, k_{0}\right)}+\frac{1}{1-\beta} \frac{P_{i}\left(0, k^{s}\right) \phi^{\prime}\left(k^{s}\right)}{P_{k}\left(0, k^{s}\right)} \sum_{\nu=1}^{T} \delta_{\nu-1}\left(\sigma_{\nu}-\beta\right) \\
& <-\frac{P_{i}\left(0, k^{s}\right)}{P_{k}\left(0, k^{s}\right)}+\frac{1}{1-\beta} \frac{P_{i}\left(0, k^{s}\right) \phi^{\prime}\left(k^{s}\right)}{P_{k}\left(0, k^{s}\right)} \sum_{\nu=1}^{T} \delta_{\nu-1}\left(\sigma_{\nu}-\beta\right)
\end{aligned}
$$

The ' $\leq$ ' sign in the second line holds due to condition (21), the ' $<$ ' sign in the third line holds due to equation (A.4). This implies that

$$
0<\frac{1}{1-\beta} \frac{P_{i}\left(0, k^{s}\right) \phi^{\prime}\left(k^{s}\right)}{P_{k}\left(0, k^{s}\right)} \sum_{\nu=1}^{T} \delta_{\nu-1}\left(\sigma_{\nu}-\beta\right)
$$

which can only hold for $\phi^{\prime}\left(k^{s}\right)>0$ because $P_{i}<0, P_{k}>0$ and the sum is negative, as $\sigma_{\nu}<\beta, \forall \nu \geq T$. However, $\phi^{\prime}\left(k^{s}\right)>0$ contradicts the assumption of an asymptotically stable steady state. As a consequence, the unique equilibrium is given by no investment of all agents.

Ad 2. The case of no investment is trivial. For $i_{i}>0$ for some $t \geq 0$ the following condition holds in the steady state:

$$
-\frac{P_{i}\left(0, k^{s}\right)}{P_{k}\left(0, k^{s}\right)}=\frac{\beta}{1-\beta}+\frac{1}{1-\beta}\left(1+\frac{P_{i}\left(0, k^{s}\right) \phi^{\prime}\left(k^{s}\right)}{P_{k}\left(0, k^{s}\right)}\right) \sum_{\nu=1}^{T} \delta_{\nu-1}\left(\sigma_{\nu}-\beta\right) .
$$

We know that $-1 \leq \phi^{\prime}\left(k^{s}\right) \leq 0$ for an asymptotically stable steady state. Thus, there exists a lower and an upper bound for the set of asymptotically stable steady states, $\underline{k}^{s}$ 
and $\bar{k}^{s}$, which are given by the following implicit equations:

$$
\begin{aligned}
& -\frac{P_{i}\left(0, \underline{k}^{s}\right)}{P_{k}\left(0, \underline{k}^{s}\right)}=\frac{\beta}{1-\beta}+\frac{1}{1-\beta}\left(1-\frac{P_{i}\left(0, \underline{k}^{s}\right)}{P_{k}\left(0, \underline{k}^{s}\right)}\right) \sum_{\nu=1}^{T} \delta_{\nu-1}\left(\sigma_{\nu}-\beta\right), \\
& -\frac{P_{i}\left(0, \bar{k}^{s}\right)}{P_{k}\left(0, \bar{k}^{s}\right)}=\frac{\beta}{1-\beta}+\frac{1}{1-\beta} \sum_{\nu=1}^{T} \delta_{\nu-1}\left(\sigma_{\nu}-\beta\right) .
\end{aligned}
$$

Note that the lower bound $\underline{k}^{s}$ corresponds to an equilibrium rule with $\phi^{\prime}\left(\underline{k}^{s}\right)=-1$ and the upper bound $\bar{k}^{s}$ corresponds to an equilibrium rule with $\phi^{\prime}\left(\bar{k}^{s}\right)=0$.

\section{A.4 Proof of Proposition 5}

Ad 1. A commitment to the ex ante optimal plan is always Pareto optimal, as the ex ante optimal plan is unique, due to the assumed curvature properties. Thus, any deviation from it would decrease the utility of agent zero (otherwise it would not have been optimal in the first place).

Ad 2. To show that the outcome of naive and sophisticated agents may be inefficient, we construct an example, for which we assume that agents discount quasi-hyperbolically, that is $T=1$ with $\sigma_{1}=\alpha \beta, 0<\alpha<1$. We further assume that condition (13) holds, which implies that neither naive nor sophisticated agents will invest although investment is ex ante optimal. Thus, the following condition holds:

$$
\frac{\beta}{1-\beta}>-\frac{P_{i}\left(0, k_{0}\right)}{P_{k}\left(0, k_{0}\right)}=-\frac{P_{i}^{0}}{P_{k}^{0}} \geq \frac{\alpha \beta}{1-\beta}
$$

To show that a Pareto improvement for naive and sophisticated agents may be possible, consider the utility effect of marginal investments $\Delta i_{0}$ and $\Delta i_{1}$ of agents zero and 1 . To keep the analysis simple we assume that all other agents do not invest. Note that the utility of all other agents increases if agents zero and 1 increase their investments. Then, the net utility effects of the investments $\Delta i_{0}$ and $\Delta i_{1}$ for agents zero and 1 are given by:

$$
\begin{aligned}
& \Delta W_{0}=\Delta i_{0}\left(P_{i}^{0}+P_{k}^{0} \frac{\alpha \beta}{1-\beta}\right)+\Delta i_{1} \alpha \beta\left(P_{i}^{0}+P_{k}^{0} \frac{\beta}{1-\beta}\right), \\
& \Delta W_{1}=\Delta i_{1}\left(P_{i}^{0}+P_{k}^{0} \frac{\alpha \beta}{1-\beta}\right)+\Delta i_{0}\left(P_{k}^{0}+P_{k}^{0} \frac{\alpha \beta}{1-\beta}\right) .
\end{aligned}
$$

According to condition (A.18), for both equations the first term is negative and the second term is positive. If agent 1 invests to such an amount that her net utility gain is zero, we derive for $\Delta i_{1}$ :

$$
\Delta i_{1}=-\Delta i_{0} \frac{P_{k}^{0}+P_{k}^{0} \frac{\alpha \beta}{1-\beta}}{P_{i}^{0}+P_{k}^{0} \frac{\alpha \beta}{1-\beta}}>0 .
$$

Inserting into $\Delta W_{0}$ and dividing by $\Delta i_{0}$ yields:

$$
\frac{\Delta W_{0}}{\Delta i_{0}}=P_{i}^{0}+P_{k}^{0} \frac{\alpha \beta}{1-\beta}-\frac{\alpha \beta\left(P_{i}^{0}+P_{k}^{0} \frac{\beta}{1-\beta}\right)\left(P_{k}^{0}+P_{k}^{0} \frac{\alpha \beta}{1-\beta}\right)}{P_{i}^{0}+P_{k}^{0} \frac{\alpha \beta}{1-\beta}} .
$$

If, for example, $P_{i}^{0}=-12, P_{k}^{0}=1, \alpha=0.5$ and $\beta=0.95$ then $\Delta W_{0} / \Delta i_{0}>0$ and, there- 
fore, a Pareto improvement can be achieved if both agents depart from the no investment equilibrium. 


\section{References}

Ackerlof, G. (1991): 'Procrastination and obedience', American Economic Review, 81: $1-19$.

AzFAR, O. (1999): 'Rationalizing hyperbolic discounting', Journal of Economic Behaviour \& Organization, 38: 245-52.

Brocas, I. AND J. D. CARRILlo (2001): 'Rush and procrastination under hyperbolic discounting and independent activities', Journal of Risk and Uncertainty, 22: 141-44.

Chichilnisky, G. (1996): 'An axiomatic approach to sustainable development', Social Choice and Welfare, 13: 231-57.

Cropper, M. And D. LAiBson (1999): 'The implications of hyperbolic discounting for project evaluation'. In Portney, P. R. And J. P. Weyant (Eds.): Discounting and Intergenerational Equity. Resources for the Future, Washington DC.

Debreu, G. (1954): 'Representation of a preference ordering by a numerical function'. In Thrall, R. M., Davis, R. L. And C.H. Coombs (Eds.): Decision Process. John Wiley, New York. Pages 159-65.

Dixit, A. K. (1992): 'Investment and hysteresis', Journal of Economic Perspectives, 6: $107-32$.

Dixit, A. K. AND R.S. Pindyck (1994): Investment under Uncertainty. Princteon University Press, Princeton.

Frederick, S., Loewenstein, G. And T. O'Donoghue (2002): 'Time discounting and time preference: A critical review', Journal of Economic Literature, 40: 351-401.

Gintis, H. (2000): 'Beyond homo economicus: evidence from experimental economics', Ecological Economics, 35: 311-22.

Gollier, C. (2002): 'Discounting an uncertain future', Journal of Public Economics, 85: $149-66$.

Groom, B., Hepburn, C., Koundouri, P. and D. Pearce (2005): 'Declining discount rates: the long and the short of it', Environmental and Resource Economics, 32: 44593.

Gruber, J. And B. Koszegi (2001): 'Is addiction rational? Theory and evidence', Quarterly Journal of Economics, 116: 1261-305.

HaRris, C. AND D. LAIBSOn (2001): 'Dynamic choices of hyperbolic consumers', Econometrica, 69: 935-57.

Heal, G. (1998): Valuing the Future: Economic Theory and Sustainability. Columbia University Press, New York.

Henderson, N. and I. Bateman (1995): 'Empirical and public choice evidence for hyperbolic social discount rates and the implications for intergenerational discounting', Environmental and Resource Economics, 5: 413-23.

KARP, L. (2005): 'Global warming and hyperbolic discounting', Journal of Public Economics, 89: 261-82.

KARP, L. (2007): 'Non-constant discounting in continuous time', Journal of Economic Theory, 132: 557-68.

www.economics-ejournal.org 
KARP, L. AND T. FUJII (2008): 'Numerical analysis of non-constant pure rate of time preference: A model of climate policy', Journal of Environmental Economics and Management, 56: 83-101.

Koopmans, T. C. (1960): 'Stationary ordinal utility and impatience', Econometrica, 28: 287-309.

LAIBSON, D. (1997): 'Golden eggs and hyperbolic discounting', Quarterly Journal of Economics, 112: 443-79.

LAIBSON, D. (1998): 'Life-cycle consumption and hyperbolic discount functions', European Economic Review, 42: 861-71.

Li, C. Z. AND K. G. LÖFGREN (2000): 'Renewable resources and economic sustainability: A dynamic analysis with heterogenous time preferences', Journal of Environmental Economics and Management, 40: 236-50.

Lind, R. C. (Ed.) (1982): Discounting for Time and Risk in Energy Policy. John Hopkins University Press, Baltimore.

O'Donoghue, T. And M. Rabin (1999): 'Doing it now or later', American Economic Review, 89: 103-24.

Pearce, D. (2003): 'Will global warming be controlled? Reflections on the irresolution of humankind'. In Pethig, R. And M. Raucher (Eds.): Challenges to the World Economy. Festschrift for Horst Siebert. Springer, Berlin.

Pearce, D., Groom, B., Hepburn, C. and P. Koundouri (2003): 'Valuing the future. Recent advances in social discounting', World Economics, 4: 121-41.

Phelps, E. S. AND R. A. Pollak (1968): 'On second-best national saving and gameequilibrium growth', Review of Economic Studies, 35: 185-99.

Portney, P. R. And J. P. Weyant (Eds.) (1999): Discounting and Intergenerational $\underline{\text { Equity. }}$ Resources for the Future, Washington DC.

Proops, J. L. R. (2001): 'The (non-) economics of the nuclear fuel cycle: an historical and discourse analysis', Ecological Economics, 39: 13-19.

RABL, A. (1996): 'Discounting of long-term costs: what would future generations prefer us to do?', Ecological Economics, 17: 137-45.

SAlanié, F. AND N. TREICH (2006): 'Over-savings and hyperbolic discounting', European Economic Review, 50: 1557-70.

Samuelson, P.A. (1937): 'A note on measurement of utility', Review of Economics Studies, 4: $155-61$.

Strotz, R. H. (1956): 'Myopia and inconsistency in dynamic utility maximization', Review of Economic Studies, 23: 165-180.

Weitzman, M. L. (1998): 'Why the far distant future should be discounted at its lowest possible rate', Journal of Environmental Economics and Management, 36: 201-8.

Wilson, P. D. (Ed.) (1996): The Nuclear Fuel Cycle: From Ore to Wastes. Oxford University Press, Oxford. 


\section{conomics}

The Open-Access, Open-Assessment E-Journal

\section{Please note:}

You are most sincerely encouraged to participate in the open assessment of this article. You can do so by either rating the article on a scale from 1 (bad) to 5 (excellent) or by posting your comments.

\section{Please go to:}

www.economics-ejournal.org/economics/journalarticles/2009-12

The Editor 